\title{
Panorama dos conteúdos de esporte adaptado nos programas de Pós-Graduação strito sensu de Educação Física no Brasil
}

\author{
Overview of the adapted sport content in stricto sensu Physical \\ Education Postgraduate Programs from Brazil
}

\begin{abstract}
Eduardo Klein Carmona1, Rafaela Bertoldi, Janice Zarpellon Mazo', Marli Hatje², Luciana Erina Palma² Universidade Federal do Rio Grande do Sul (UFRGS), Porto Alegre, RS, Brasil.

universidade Federal de Santa Maria (UFSM), Santa Maria, RS, Brasil.
\end{abstract}

Recebido em: 03/05/2016 / Aceito em: 22/06/2016

eduardok.carmona@hotmail.com

\section{RESUMO}

Objetivo: apresentar um panorama dos conteúdos relativos à atividade física e ao esporte adaptado nos cursos de Pós-Graduação strito sensu, em Educação Física no Brasil. Método e Resultados: foram analisadas as informações que resultaram da avaliação dos PPGs disponibilizados pela CAPES e nos websites de cada curso no ano de 2013. Utilizaram-se as informações das áreas de concentração, linhas de pesquisa e disciplinas dos 29 PPGs na área de Educação Física existentes no Brasil. Destes, apenas o PPG da Unicamp apresenta uma área de concentração e duas linhas de pesquisa destinadas a promover estudos relacionados às pessoas com deficiência. A UNB oferece uma linha de pesquisa específica para estudos sobre pessoas com deficiência. Quanto às disciplinas, 11 cursos abordam a temática, sendo grande parte concentrados na UNICAMP. Considerações finais: os dados revelam que os conteúdos sobre atividade física adaptada e esporte adaptado ainda carecem de mais espaço na pós-graduação stricto sensu.

Palavras-chave: Atividade Física Adaptada; Esporte Adaptado; Pessoas com Deficiência; Programas de Pós-Graduação.

\section{ABSTRACT}

Objective: introduce an overview of contents concerning adapted physical activity and sports in stricto sensu Physical Education Postgraduate Programs from Brazil. Method and Results: for this purpose, information from the Postgraduate Programs evaluation, made available by CAPES and in each program websites in 2013, were analyzed. The information provided by each field of study, line of research e discipline of the 29 different Postgraduate Programs in the Physical Education area current in Brazil were used. Among them, only the Unicamp Postgraduate Program has a field of study and two lines of research directed to promote studies related to people with disabilities. The UNB offers one specific research field to develop studies about people with disabilities. About the disciplines, 11 Programs approach this theme, especially in UNICAMP. Closing remarks: the information revealed that the contents about adapted physical activity and sports are barely studied in the stricto sensu Postgraduate scene.

Keywords: Adapted Physical Activity; Disability Sport; People with Disability; Postgraduate Programs.

\section{INTRODUÇÃO}

Os periódicos acadêmicos são os grandes responsáveis pela divulgação imediata do conhecimento científico mundial. ${ }^{1}$ Todavia, a produção de conhecimentos está fortemente atrelada aos programas de pós-graduação (PPGs) stricto sensu, que oferecem curso de mestrado e/ou doutorado. Os PPGs são avaliados pela Coordenação de Aperfeiçoamento de Pessoal de Nível Superior (CAPES) e, atualmente, são divididos em 48 áreas distintas. ${ }^{2}$

A Educação Física, por sua vez, está compreendida em uma área ampla, juntamente com a Fisioterapia, Fonoaudiologia e Terapia Ocupacional, denominada de área 21. De acordo com o Relatório de Avaliação Trie- 
nal da CAPES, publicado em 2013, a área 21 está em expansão, tendo aumentado o número de programas, bem como os cursos de doutorado entre os programas.

Os programas dessa área desenvolvem estudos de diversas naturezas, promovendo desde pesquisas da subárea da biodinâmica, até investigações pedagógicas e socioculturais, o que, ao mesmo tempo, demonstram e reforçam a amplitude e abrangência do campo de Educação Física. ${ }^{3}$ Contudo, o ponto comum entre todos os PPGs e as investigações desenvolvidas por seus discentes corresponde à relação direta com o movimento humano nos diversos âmbitos. Desta forma, populações e grupos com distintas características biológicas e sociais são abarcadas nessas investigações, como, por exemplo, as pessoas com deficiência.

As pessoas com deficiência configuram um tipo de grupo unido por diferenças físico-motoras, sensoriais ou intelectuais consideradas atípicas para os padrões do ser humano. Brumer, Pavei e Mocelin, ${ }^{4}$ por sua vez, afirmam que a deficiência é uma construção sócio-histórica-cultural que busca agrupar e ao mesmo tempo diferençar as pessoas por características comuns e outras nem tão comuns de alguns seres humanos. De acordo com o Censo 2010, no Brasil, aproximadamente 45,6 milhões de pessoas declararam ter ao menos um tipo de deficiência, o que corresponde a $23 \%$ da população do país. ${ }^{5}$ Tais dados contribuem identificar as pessoas com deficiência como um grupo que merece atenção da ciência brasileira, isso nas diversas áreas do conhecimento e incluindo a da Educação Física.

Historicamente, no campo da Educação Física brasileira, pouca atenção essa população recebeu no âmbito das pesquisas científicas e por consequência nos PGGs da área, ${ }^{6}$ visto a ligação marcante entre as produções científicas e a pós-graduação no país. A população com deficiência sofreu com a exclusão ou o aniquilamento durante muito tempo e essas mazelas históricas fizeram com que os deficientes não fossem público-alvo de investigações relacionadas ao movimento e a atividade física. ${ }^{7}$ Diante deste cenário, o presente estudo tem por objetivo apresentar um panorama dos conteúdos relativos à atividade física e ao esporte adaptado nos cursos de Pós-Graduação em Educação Física no Brasil.

\section{MÉTODO}

Nesta investigação, buscamos, por meio de dados da avaliação dos PPGs no Brasil fornecidos e disponibilizados pela CAPES e nos websites de cada programa da área 21, (Educação Física), identificar se ocorreu ou não o desenvolvimento de conteúdos acerca das pessoas com deficiência. Para tanto, as informações selecionadas foram as áreas de concentração, as linhas de pesquisa e as disciplinas oferecidas pelos PPGs. Cabe referir que a área de concentração expressa a tradição de uma instituição, de seus docentes e do próprio PPG, além de indicar a área do conhecimento e as especialidades da produção de conhecimento e da formação dos dis centes. As linhas de pesquisa refletem a especificidade da produção de conhecimento do programa nas áreas de concentração. Por fim, as disciplinas manifestam os conhecimentos basilares para a formação dos discentes e representam a tradição histórica do programa.

Atualmente são reconhecidos e recomendados 52
PPGs na área 21, com cursos de mestrado acadêmico, doutorado acadêmico e/ou mestrado profissional, sendo 29 vinculados diretamente à Educação Física. ${ }^{2}$ No quadro 1, identificamos os PPGs, suas instituições de origem, os estados onde estão localizados e o(s) nível(is) oferecido(s) por cada um.

Quadro 1 - PPGs da área 21 vinculados diretamente à Educação Física.

\begin{tabular}{|c|c|c|c|c|c|}
\hline \multirow[t]{2}{*}{ PROGRAMA } & \multirow[t]{2}{*}{ IES } & \multirow[t]{2}{*}{ UF } & \multicolumn{3}{|c|}{ NÍVEL } \\
\hline & & & M & D & $\mathbf{P}$ \\
\hline Ciências da Atividade Física & UNIVERSO & RJ & $\mathrm{x}$ & - & - \\
\hline Ciências da Atividade Física & USP & SP & $\mathrm{x}$ & - & - \\
\hline Ciências da Motricidade & UNESP/RC & $\mathrm{SP}$ & $x$ & $x$ & - \\
\hline Ciências do Esporte & UFMG & MG & $\mathrm{x}$ & $x$ & - \\
\hline Ciências do Exercício e do Esporte & UERJ & $\mathrm{RJ}$ & $\mathrm{x}$ & $\mathrm{x}$ & - \\
\hline Ciências do Movimento Humano & UDESC & $\mathrm{SC}$ & $\mathrm{x}$ & $\mathrm{x}$ & - \\
\hline Ciências do Movimento Humano & UFRGS & $\mathrm{RS}$ & $x$ & $x$ & - \\
\hline Ciências do Movimento Humano & UNICSUL & $\mathrm{SP}$ & $x$ & $x$ & - \\
\hline Ciências do Movimento Humano & UNIMEP & SP & $\mathrm{x}$ & $\mathrm{x}$ & - \\
\hline Educação Física & FESP/UPE & $\mathrm{PE}$ & $\mathrm{x}$ & $\mathrm{x}$ & - \\
\hline Educação Física & FUFSE & SE & $\mathrm{x}$ & - & - \\
\hline Educação Física & UCB & DF & $\mathrm{x}$ & $\mathrm{x}$ & - \\
\hline Educação Física & UEL/UEM & $\mathrm{PR}$ & $\mathrm{x}$ & $\mathrm{x}$ & - \\
\hline Educação Física & UFES & $\mathrm{ES}$ & $x$ & $x$ & - \\
\hline Educação Física & UFMT & MT & $x$ & - & - \\
\hline Educação Física & UFPEL & $\mathrm{RS}$ & $\mathrm{x}$ & $x$ & - \\
\hline Educação Física & UFPR & PR & $\mathrm{x}$ & $\mathrm{x}$ & - \\
\hline Educação Física & UFRJ & $\mathrm{RJ}$ & $\mathrm{x}$ & - & - \\
\hline Educação Física & UFRN & RN & $\mathrm{x}$ & - & - \\
\hline Educação Física & UFSC & $\mathrm{SC}$ & $\mathrm{x}$ & $x$ & - \\
\hline Educação Física & UFSM & RS & $x$ & - & - \\
\hline Educação Física & UFTM & MG & $x$ & - & - \\
\hline Educação Física & UFV/UFJF & MG & $x$ & - & - \\
\hline Educação Física & UNB & DF & $\mathrm{x}$ & $\mathrm{x}$ & - \\
\hline Educação Física & UNICAMP & $\mathrm{SP}$ & $\mathrm{x}$ & $\mathrm{x}$ & - \\
\hline Educação Física & UNIMEP & $\mathrm{SP}$ & $\mathrm{x}$ & - & - \\
\hline Educação Física & USJT & $\mathrm{SP}$ & $\mathrm{x}$ & $x$ & - \\
\hline Educação Física e Esporte & USP & $\mathrm{SP}$ & $x$ & $x$ & - \\
\hline $\begin{array}{l}\text { Exercício Físico na Promoção da } \\
\text { Saúde }\end{array}$ & UNOPAR & PR & - & - & $x$ \\
\hline
\end{tabular}

Fonte: Adaptado da Relação de Cursos Recomendados e Reconhecidos da CAPES

\section{RESULTADOS E DISCUSSÃO}

A partir do quadro1, podemos identificar que os PPGs da área da Educação Física se concentram majoritariamente nas regiões sudeste e sul do país, tendo destaque para o estado de São Paulo, com oito programas. Do total de 29 PPGs da área da Educação Física no Brasil, o único que apresenta uma área de concentração destinada diretamente a promover estudos relacionados às pessoas com deficiência é o PPG em Educação Física da Universidade Estadual de Campinas (UNICAMP). Denominada como Atividade Física Adaptada, esta área de concentração se propõe a investigar "aspectos vinculados à atividade física, esporte, imagem corporal e qualidade de vida dos grupos com necessidades especiais (pessoas com deficiência, idosos, hipertensos, entre outros) ou expostos a possíveis situações de dano ao bem estar". ${ }^{8}$ Esta área de concentração apresenta duas linhas de pesquisa: Atividade Física para Pessoas com Deficiências, a qual é exclusiva a investigações 
sobre essa população; e Atividade Física para Grupos Especiais, destinado a outros grupos como, por exemplo, idosos e doentes crônicos.

Outro PPG que possui uma linha de pesquisa relacionada é o de Educação Física da Universidade de Brasília (UNB), a qual é nomeada “Exercício Físico e Reabilitação para Populações Especiais. Esta linha de pesquisa "investiga as adaptações fisiológicas e biomecânicas propiciadas pelo exercício e pela atividade física nas suas diferentes formas de intervenção em idosos, diabéticos, obesos, cardiopatas, portadores de necessidades especiais, entre outros". 8 Ainda, cabe destacar que a respectiva linha de pesquisa utiliza uma nomenclatura defasada para fazer referência às pessoas com deficiência, ou seja, a expressão Portadores de Necessidades Especiais (PNE's).

Por sua vez, a linha de pesquisa, Atividade Física para Pessoas com Deficiências do PPG da UNICAMP busca investigar "aspectos relacionados à atividade física, esporte, imagem corporal e qualidade de vida de pessoas com deficiência física, intelectual e sensorial (visual e auditiva)". Desta forma, diferentemente da linha de pesquisa da Educação Física da UNB, esta apresenta um universo empírico mais específico ao estudo acerca das pessoas com deficiência. O PPG da UNICAMP, ao longo dos anos, produziu um número mais significativo do ponto de vista quantitativo de dissertações e teses em relação ao PPG da UNB. Da mesma forma, a publicação de artigos em periódicos pela UNICAMP também foi superior. Talvez, esta situação esteja relacionada ao tempo de existência de cada programa.

Com relação às disciplinas dos PPGs sobre a temática com deficiência, localizamos 11 disciplinas distintas oferecidas por sete universidades diferentes,

Quadro 2 - Disciplinas/conteúdos dos PPGs relacionados às pessoas com deficiência.

\begin{tabular}{|c|c|c|}
\hline NOME DA DISCIPLINA & EMENTA & PPG \\
\hline $\begin{array}{l}\text { Atividade física e aptidão } \\
\text { física relacionada à saúde de } \\
\text { pessoas com deficiência }\end{array}$ & $\begin{array}{l}\text { Evolução histórica do conceito de deficiência. Particularidades da elaboração de programas de exercícios } \\
\text { voltados à saúde de pessoas com deficiência visual, auditiva, motora, intelectual e múltipla. Comportamen- } \\
\text { tos voltados à saúde e procedimentos de medidas e avaliação das principais variáveis da aptidão física de } \\
\text { pessoas com deficiência. Tendências atuais de pesquisa na área da aptidão física relacionada à saúde de } \\
\text { pessoas com deficiência. }\end{array}$ & UEL/UEM \\
\hline Atividade física adaptada & $\begin{array}{l}\text { Caracterização da pessoa com deficiência. Introdução à atividade física adaptada e o processo inclusivo. In- } \\
\text { tervenções de promoção da atividade física e saúde. Processo inclusivo atual; Deficiência: o que é, quantos } \\
\text { são e tipos de deficiência? Atividade física, promoção da saúde e qualidade de vida: aspectos individuais, } \\
\text { culturais e ambientais; Benefícios da atividade física adaptada: (esportivas, aquáticas, recreativas, dança } \\
\text { e lazer); Mensuração de atividade física; Estudos em atividade física em diferentes deficiências; Atividade } \\
\text { física na prevenção e tratamento de doenças; Atividade física, saúde e estilo de vida de pessoas com } \\
\text { deficiência; Intervenções de promoção da atividade física e saúde; Mudança dos paradigmas: Futuro da } \\
\text { Educação Física adaptada. }\end{array}$ & UFPEL \\
\hline Atividade física adaptada & $\begin{array}{l}\text { Análise de modelos de intervenção em portadores de deficiência, na perspectiva da implementação de pro- } \\
\text { gramas de atividade física para reabilitação física e mental, com ênfase no desenvolvimento biopsicossocial. }\end{array}$ & UFRN \\
\hline Atividade motora adaptada & $\begin{array}{l}\text { Estudo de modelos de intervenção em pessoas portadoras de necessidades educativas especiais, na área } \\
\text { da reabilitação física e mental, com ênfase no desenvolvimento biopsicossocial. Neste sentido, a disciplina } \\
\text { busca uma prática pedagógica junto aos profissionais na área da saúde e educação, proporcionando uma } \\
\text { interface com diversas áreas do conhecimento. }\end{array}$ & UNB \\
\hline $\begin{array}{l}\text { Desenvolvimento sensório- } \\
\text { motor e perceptivo-motor de } \\
\text { populações especiais }\end{array}$ & $\begin{array}{l}\text { Este curso oferece um exame dos conceitos e abordagens do desenvolvimento com o objetivo de en- } \\
\text { tender mecanismos do comportamento motor e perceptuais, e sensório-motores de populações especiais. } \\
\text { Particularmente discute fatores críticos que influenciam o processo de desenvolvimento sensório-motor } \\
\text { e perceptivo-motor ao longo da vida em: indivíduos portadores de deficiência mental, paralisia cerebral, } \\
\text { autismo e outras condições psicobiológicas atípicas. Serão analisados estudos explorando mecanismos } \\
\text { adaptativos de populações especiais na: integração sensório-motora, percepção do espaço de movimento, } \\
\text { da forma e estrutura dos objetos e ambiente, e outras temáticas relacionadas à percepção-ação. Os alunos } \\
\text { terão experiências práticas observação de casos dos quais terão oportunidades de aplicar os conceitos } \\
\text { discutidos em classe. }\end{array}$ & UNESP \\
\hline $\begin{array}{l}\text { Abordagem de pesquisa em } \\
\text { atividade física e adaptação }\end{array}$ & Métodos e técnicas nas linhas de pesquisas da área de concentração. & UNICAMP \\
\hline $\begin{array}{l}\text { Estudos avançados em } \\
\text { esporte adaptado }\end{array}$ & $\begin{array}{l}\text { Estudo do treinamento em atletas e praticantes de esportes adaptado. Estudos em modalidades esporti- } \\
\text { vas adaptadas. Estudos em modalidades esportivas paraolímpicas. Recursos tecnológicos. Classificação } \\
\text { funcional. }\end{array}$ & UNICAMP \\
\hline $\begin{array}{l}\text { Estudos independentes em } \\
\text { educação física adaptada }\end{array}$ & Leituras e atividades independentes realizadas em Educação Física Adaptada. & UNICAMP \\
\hline $\begin{array}{l}\text { Processos de avaliação } \\
\text { motora em educação física } \\
\text { adaptada }\end{array}$ & $\begin{array}{l}\text { Estudo dos aspectos gerais da avaliação para pessoas com deficiência. Técnicas, instrumentos, protocolos e } \\
\text { padronização de medidas. Adaptações e ajustes de testes para diferentes grupos. }\end{array}$ & UNICAMP \\
\hline $\begin{array}{l}\text { Tópicos em estudos da } \\
\text { atividade física adaptada }\end{array}$ & $\begin{array}{l}\text { Abordagem de tópicos escolhidos de comum acordo entre o docente e os alunos. Estudo de temas sobre } \\
\text { correlações anatomo-fisiológicas em distúrbios do movimento e a pessoa com deficiência. }\end{array}$ & UNICAMP \\
\hline $\begin{array}{l}\text { Tópicos em estudos da } \\
\text { atividade física, adaptação } \\
\text { e saúde }\end{array}$ & $\begin{array}{l}\text { Abordagem de tópicos escolhidos de comum acordo entre o docente e os alunos. Estudo de temas sobre } \\
\text { educação física especial, esportes adaptados, nutrição, } 3^{\mathrm{a}} \text { idade, doenças crônicas, avaliação e elaboração } \\
\text { de programas dirigidos à promoção da saúde e à qualidade de vida. }\end{array}$ & UNICAMP \\
\hline
\end{tabular}

Fonte: Quadro elaborado a partir do caderno de indicadores dos PPGs da CAPES. 
sendo três instituições federais e quatro estaduais. No entanto, não obtivemos informações sobre o número de alunos matriculados nas respectivas disciplinas, um dado que se considera relevante para verificar a formação docente. Nesta direção, outro dado que poderia contribuir para uma apreciação das respectivas disciplinas é a obrigatoriedade ou não no currículo. Todavia, estas informações ainda não foram adquiridas, embora solicitadas aos PPGs. O quadro abaixo representa o nome das disciplinas, seus respectivos programas e suas ementas, conforme consta nas páginas eletrônicas dos PPGs.

O quadro 2, além de demonstrar que as disciplinas relacionadas às pessoas com deficiências se concentram no programa da UNICAMP, devido à tradição da instituição, ao mesmo tempo, também reforça que os conteúdos acerca dessa população são incipientes e pouco estudados nos PPGs da área. De acordo com as ementas, as disciplinas se propõem a conceituar aspectos relacionados ao universo das pessoas com deficiência, discutir as possíveis intervenções da Educação Física com a população, bem como abordam aspectos acerca de atividades físicas e saúde. As disciplinas do programa da UNICAMP aprofundam também outras temáticas acerca das pessoas com deficiência, como, por exemplo, esportes adaptados.

No campo da Educação Física no Brasil, a demanda por conteúdos acerca das pessoas com deficiência começou a ser discutida juntamente com os debates sobre a reforma curricular dos cursos de graduação em Educação Física na década de 1980. Com o Parecer $n^{\circ}$ 215 de 1987 e com a Resolução $n^{\circ}$ 03, de 16 de junho de 1987, houve um relativo avanço nesta questão, pois o próprio indicava a inserção da Educação Física adaptada nos currículos. ${ }^{9,10}$ As disciplinas específicas, portanto, devem "permitir que o graduando compreenda as características da pessoa com deficiência e como isso influencia a sua prática de atividade física, levando o futuro professor de Educação Física a ampliar suas possibilidades de atuação, permitindo a inclusão social em suas aulas"11 (p. 584).

No entanto, mesmo com a obrigatoriedade, o processo de inserção nos cursos de Educação Física foi demorado, pois não havia professores capacitados para ministrarem os conteúdos nos cursos. Como um modo de impulsionar este campo do conhecimento na área da Educação Física, promoveu-se, principalmente, no decorrer da década de 1990, diversos cursos de pós-graduação lato sensu sobre Educação Física adaptada com o intuito de formar recursos humanos. Nesse período, grande parte dos professores dos cursos de especialização eram oriundos de outras áreas e/ou de outros países para ministrar as aulas de forma intensiva12. A Universidade Federal de Uberlândia (UFU) tem um papel de destaque na promoção, desde a década de 1980, de cursos lato sensu, enfocando atividades físicas e esportivas para pessoas com deficiência.

Paralelamente ao processo de formação de professores para o ensino superior e inserção de disciplinas acerca das pessoas com deficiência nos cursos de graduação em Educação Física, ainda na década de 1990, foi criada a Sociedade Brasileira de Atividade Motora Adaptada (SOBAMA). A entidade visa agregar estudiosos da área de atividade motora adaptada, apoiar e incentivar o desenvolvimento técnico-científico. A SOBAMA realiza diversas atividades para promover a área, como, por exemplo, congressos, cursos e publicações. ${ }^{13}$

\section{CONSIDERACQÕES FINAIS}

As iniciativas mencionadas acima foram relevantes para a consolidação da área de concentração "Atividade Física Adaptada" no PPG em Educação Física da UNICAMP. Esta instituição de ensino superior é uma das precursoras na formação de mestres e doutores para atuar com pessoas com deficiência no Brasil. Todavia, não podemos esquecer as disciplinas oferecidas em outros PPGs, as quais revelam certa tradição e interesse pela área dos professores que as ministram. Além disso, como evidencia a publicação de Silva e Araújo (2012), houve um crescimento da pesquisa em atividade motora adaptada.

Os dados apresentados neste estudo foram publicados em 2013, contudo apresentam um cenário relativo ao triênio anterior (2010-2011-2012). No período de 2013, até o tempo presente, provavelmente outros cursos de pós-graduação passaram a contemplar em suas linhas de pesquisa o esporte adaptado e o esporte paralímpico. Este é o caso do Programa de Pós-Graduação em Ciências do Movimento Humano (PPGCMH) que na linha de concentração “Representação Social do Movimento Humano", tem uma linha de pesquisa individual que privilegia estudos sobre as pessoas com deficiência.

O movimento de inserção de novas disciplinas voltadas às temáticas relacionadas à prática esportiva por pessoas com deficiência se desenvolveu como um reflexo de uma maior inclusão desta população na sociedade atual. Possivelmente, isto ocorreu também em razão do desempenho do Brasil nas últimas edições dos Jogos Paralímpicos, que oportunizou a essas pessoas uma maior visibilidade. Além disso, a escolha do Brasil para sede dos Jogos Olímpicos e Paralímpicos de 2016, também tem contribuído para mais divulgação sobre o esporte e sua prática pelas pessoas com deficiência. Tais fatos impulsionaram a adaptação da Educação Física a essa nova realidade, pois é necessária a formação de profissionais para atuarem com esse público específico.

\section{REFERÊNCIAS}

1. Job Ivone. Identificação das revistas científicas brasileiras da área de educação física e esportes. Caderno de Educação Física - UNIOESTE, (Marechal Cândido Rondon) 2009;8(14):9-18.

2. CAPES. Educação Física. Coordenação de Aperfeiçoamento de Pessoal de Nível Superior. Brasília. Disponível em: <http://www.capes.gov.br/component/content/article/44avaliacao/4666-educacao-fisica> Acesso em: 15 abril 2016.

3. Bracht V. Desafios e dilemas da Pós-graduação em Educação Física: conhecimento e especificidade. In: Rechia $\mathrm{S}$ et al.(Orgs). Dilemas e Desafios da Pós-Graduação em Educação Física. ljuí: Ed. Unijuí 2015;109-124.

4. Brumer A, Pavei K, Mocelin D. G. Saindo da "escuridão": perspectivas da inclusão social, econômica, cultural e política dos portadores de deficiência visual em Porto Alegre. Sociologias 2004;6(11):300-327. 
5. Oliveira LMB. Cartilha do Censo 2010 - Pessoas com Deficiência. Brasília; SDH-PR/SNPD, 2012.

6. Silva RF, Araujo PF. Os caminhos da pesquisa em atividade motora adaptada. São Paulo; Phorte. 2012.

7. Rechineli A, Porto ETR, Moreira WW. Corpos deficientes, eficientes e diferentes: uma visão a partir da educação física. Rev Bras Educ Espec 2008;14(2):293-310.

8. Atividade Física Adaptada. Áreas de concentração. Programa de Pós-Graduação em Educação Física da UNICAMP. Campinas. Disponível em:< http://www.fef.unicamp.br/fef/ posgraduacao> Acesso em: 28 abr. 2016

9. Ribeiro SM, Araújo PF. A formação acadêmica refletindo na expansão do desporto adaptado: uma abordagem brasileira. Rev Bras Ciênc Esporte. (Campinas) 2004;25(3): 57-69.
10. Araújo PF. Desporto Adaptado no Brasil. São Paulo; Phorte, 2011.

11. Ferreira E, Lopes RGB, Ferreira R, Nista-Piccolo VL. Um olhar sobre a educação física adaptada nas universidades públicas paulistas: atividades obrigatórias e facultativas. Rev Educ Fís. UEM, (Maringá) 2013;24(4):581-595.

12. Lima SR. Cursos de especialização em Educação Física e Esportes Adaptados: onde estão os egressos? 1998. 152f. Dissertação (Mestrado) - Mestrado em Educação, Universidade Federal de Uberlândia, Uberlândia, 1998.

13. Sobama. O que é Sobama?. Sociedade Brasileira de Atividade Motora Adaptada. Rio Claro. Disponível em: <http://www. rc.unesp.br/ib/efisica/sobama/sobamaorg/inicio.htm> Acesso em: 20 abril 2016.

Como citar: CARMONA, Eduardo Klein et al. Panorama dos conteúdos de esporte adaptado nos programas de pós-graduação strito sensu de Educacão Física no Brasil. Cinergis, Santa Cruz do Sul, v. 17, n. 2, jun. 2016. ISSN 2177-4005. Disponível em: <https://online.unisc.br/seer/index.php/cinergis/article/view/7515>. Acesso em: 04 jul. 2016. doi:http://dx.doi. org/10.17058/cinergis. v17i2. 7515 . 